\title{
Correlation between attending physician's empathy and readmission rate in Internal Medicine
}

\author{
Francesco Bruggi ${ }^{1}$, Francesco Falaschi ${ }^{2}$, Antonio Di Sabatino ${ }^{1}$, Paola Preti ${ }^{1}$, Carmine \\ Tinelli $^{2}$, Dominica Dell'Aera ${ }^{2}$, Alessandra Martignoni ${ }^{2}$, and Stefano Perlini ${ }^{1}$ \\ ${ }^{1}$ Università degli Studi di Pavia \\ ${ }^{2}$ Fondazione IRCCS Policlinico San Matteo
}

May 6, 2020

\begin{abstract}
IIntroduction Physician empathy has been correlated with several outcomes in outpatient settings, demonstrating better prognosis in patients followed by highly empathetic doctors. Aim To correlate empathy of Internal Medicine ward physicians with readmission rates of patients they took care of. Methods We extracted readmission data of all patients discharged from Internal Medicine wards at our hospital between January 1st, 2011 and May 31st, 2017. Patients discharged to other institutions (nursing homes, rehabilitation units, other hospitals) were excluded. The 30-day readmission rate was recorded for each doctor. We measured the empathy of physicians who discharged more than 100 patients during that period, using the Jefferson Scale of Empathy (JSE) and the Empathy Components Questionnaire (ECQ). Correlation between empathy scores and readmission rate was weighted for the number of patients followed by each physician and adjusted for sex, age and relative cost weight of patients, as well as the doctor's age. The same correlation was calculated in the subgroup of index patients discharged with Diagnosis Related Group 127 (DRG 127, Heart Failure and Shock). Results A total of 4280 index discharge events were identified, 383 of which (8.9\%) were readmitted within 30 days. JSE scores were found out to be inversely correlated with readmission rates (coefficient $-0.027, \mathrm{R} 2$ 0.181, $\mathrm{p}<0.001$ ) whereas ECQ correlation was not significant after adjustment. A significant inverse correlation was also observed considering only the DRG 127 patients, with both Empathy scales (JSE and ECQ, coefficient -0.032 and $-0.098, \mathrm{R} 20.303$ and $0.326, \mathrm{p}=0.050$ and $\mathrm{p}<0.001$, respectively). Conclusion Empathy of Internal Medicine physicians correlates with readmission rates of the patients they cared for, especially heart failure patients for whom this is a relevant outcome.
\end{abstract}

\section{AUTHORS}

Francesco Bruggi ${ }^{1}$, Francesco Falaschi ${ }^{2}$, Antonio Di Sabatino ${ }^{1,3}$, Paola Stefania Preti $^{4}$, Carmine Tinelli $^{5}$, Dominica Dell'Aera ${ }^{2}$, Alessandra Martignoni ${ }^{2}$, Stefano Perlini ${ }^{1,6}$

TITLE

Correlation between attending physicians' empathy and readmission rate in Internal Medicine.

RUNNING TITLE

Phisicians' empathy and readmission rate

AFFILIATIONS

${ }^{1}$ Facoltà di Medicina e Chirurgia, Università degli Studi di Pavia, Italia

${ }^{2}$ Medicina Generale 2, Fondazione IRCCS Policlinico San Matteo, Pavia, Italia

${ }^{3}$ Medicina Generale 1, Fondazione IRCCS Policlinico San Matteo, Pavia, Italia 
${ }^{4}$ Dipartimento di Medicina Interna e Terapia Medica, Università degli Studi di Pavia, Italia

${ }^{5}$ Servizio di Epidemiologia Clinica e Biometria, Fondazione IRCCS Policlinico San Matteo, Pavia, Italia

${ }^{6}$ Pronto Soccorso Accettazione, Fondazione IRCCS Policlinico San Matteo, Pavia, Italia

ADDRESS FOR ALL AUTHORS

Segreteria Medicina Generale 2, Viale Camillo Golgi 19, 27100 Pavia, Italia

CORRESPONDING AUTHOR

Francesco Bruggi, f.bruggi@gmail.com; (+39) 3331037126

\section{KEYWORDS}

Empathy, readmission, heart failure, Internal Medicine.

\section{ACKNOWLEDGEMENTS OF FUNDING OR OTHER SUPPORT}

No fundings or other special grants were used to collect the data and to write the article. We wish to thank the Jefferson University (Philadelphia) in the person of Professor Vittorio Maio for kind concession of free use of Jefferson Scale of Empathy anf Funda Muge Topuz (Direzione Scientifica, Fondazione IRCCS Policlinico San Matteo) for the revision of the manuscript

\section{DISCLOSURES}

None for all authors

\section{ABSTRACT}

Introduction

Physician empathy has been correlated with several outcomes in outpatient settings, demonstrating better prognosis in patients followed by highly empathetic doctors.

Aim

To correlate empathy of Internal Medicine ward physicians with readmission rates of patients they took care of.

Methods

We extracted readmission data of all patients discharged from Internal Medicine wards at our hospital between January $1^{\text {st }}, 2011$ and May $31^{\text {st }}, 2017$. Patients discharged to other institutions (nursing homes, rehabilitation units, other hospitals) were excluded. The 30-day readmission rate was recorded for each doctor.

We measured the empathy of physicians who discharged more than 100 patients during that period, using the Jefferson Scale of Empathy (JSE) and the Empathy Components Questionnaire (ECQ).

Correlation between empathy scores and readmission rate was weighted for the number of patients followed by each physician and adjusted for sex, age and relative cost weight of patients, as well as the doctor's age. The same correlation was calculated in the subgroup of index patients discharged with Diagnosis Related Group 127 (DRG 127, Heart Failure and Shock).

Results

A total of 4280 index discharge events were identified, 383 of which (8.9\%) were readmitted within 30 days. JSE scores were found out to be inversely correlated with readmission rates (coefficient $-0.027, \mathrm{R}^{2} 0.181$, $\mathrm{p}<0.001$ ) whereas ECQ correlation was not significant after adjustment. A significant inverse correlation was also observed considering only the DRG 127 patients, with both Empathy scales (JSE and ECQ, coefficient -0.032 and $-0.098, \mathrm{R}^{2} 0.303$ and $0.326, \mathrm{p}=0.050$ and $\mathrm{p}<0.001$, respectively). 
Conclusion

Empathy of Internal Medicine physicians correlates with readmission rates of the patients they cared for, especially heart failure patients for whom this is a relevant outcome.

\section{What is already known about this topic}

- Physician's Empathy is traditionally considered and important tool of medical profession but there is only little evidence of its effects on relevant clinical outcomes.

- Empathy of physicians has been correlated with patient outcomes mainly in Family Practice but not in Hospital settings.

\section{What does this article add?}

- Empathy of physicians working in an Internal Medicine ward appears inversely correlated to 30-days readmission rate of the patients they cared of

- This remains true in the big subgroup of patients with heart failure, a disease in which adherence to treatment is considered important

\section{INTRODUCTION}

The idea of the importance of an empathic patient-doctor relationship is deeply rooted in medical tradition[1] and teaching, but is surprisingly supported by only scanty evidence.[2]

Between 2011 and 2012, two studies were carried out to demonstrate the fact that the empathy of family doctors can improve the clinical outcome of patients on strong endpoints. Those studies were limited to Family practice and included only Diabetic patients, demonstrating lower glycated hemoglobin and lower rates of hospitalization for metabolic decompensation in patients of higher empathy doctors. $[3,4]$

Currently, the number of studies supporting the importance of physician's empathy is limited and doesn't focus on patient outcomes, but on patient's and physician's satisfaction. [5-12] Furthermore, the clinical impact of empathy in a hospital setting has never been studied. This actual gap of knowledge contrasts with the great importance that is traditionally credited to the doctor-patient relationship at the patient bedside.[13]

We therefore aimed to measure the clinical impact of physician's Empathy on the outcome of their patients in the Internal Medicine departments in our hospital.

To this end, we have considered the correlation between the physician's Empathy and the readmission rate of his patients in the 30 days following discharge, one of the most used indicators of hospitalized patients' outcome.[14-18] We measured the empathy of the physicians with validated self-administered scales. We obtained the readmission rate data through a combined analysis of the Emergency Room (ER) and Hospital Discharge Form (HDF) databases.

Since the readmission rate is probably influenced by other variables contained in the HDF database, we have measured these effects to control their interference on the correlations under study.

\section{METHODS AND MATERIALS}

Participation was asked to all the doctors of the Istituto di Ricovero e Cura a Carattere Scientifico (IRCCS) Policlinico "San Matteo" Foundation, who had worked in Internal Medicine wards in the years 2013-2017.

Any doctor who had filled the HDF relating to any hospitalization in Internal Medicine in the index period was considered as the discharging physician of that index case. We decided to use the doctor filling up the HDF, rather than the doctor signing the Hospital Discharge Letter (HDL), after a brief analysis of a convenience sample of three hundred HDF and HDL. In the rare cases of discrepancy, the doctor in charge of the HDF (rather than the one signing HDL) was invariably the one who had really taken care of the patient for the longest part of the hospitalization and considered himself responsible for all the effects of the case. 
Subsequently we excluded doctors who had made less than 100 ordinary discharges during the period under analysis, to have reliable readmission rates on a sufficient number of patients.

Ordinary discharge was defined as the patient's return to his usual residence: deaths, voluntary discharges, transfers to another hospital, rehabilitation transfers, Hospice transfers or discharges to Nursing home were therefore excluded, in attempt to maximally reduce the influence of other doctors' contacts on the readmission event.

Two different self-administered questionnaires were used to measure empathy in all participating physicians:

1. The Jefferson Scale of Empathy (JSE) in its official Italian version, a tool validated for use on health professionals, whose use has been kindly granted by Jefferson University (Philadelphia, Pennsylvania, USA) that holds all the rights.[2, 19]

2. The Empathy Components Questionnaire (ECQ), a validated tool for the measurement of empathy in the general population that is freely available, which we have translated into Italian specifically for this study.[20]

Both questionnaires assign higher score to higher levels of empathy. The JSE consists of 20 questions and the ECQ consists of 27 questions. For both questionnaires, the answers are given through a Likert scale ranging from 1 to 7 points for the JSE and 1 to 4 points for the ECQ.

The maximum score of the JSE is 140 points and that of the ECQ is 108 points.

Empathy is a matter potentially subject to various types of response bias: acquiescence bias and social desirability bias are the heaviest. Therefore, it was decided to ensure anonymity to the doctors who completed the questionnaires.[21]

The mechanism used to anonymize involved a guarantor (CT, the statistician of the study), who was the only person able to correlate numerical codes on the questionnaires and the names of the doctors involved in the study.

The enrollment of the doctors took place through sealed envelopes containing the coded questionnaires. The envelopes contained a print that summarized the aims of the study and the anonymization mechanism, formally asking for participation and the informing about possible publications of the data. The return by each doctor of completed questionnaires was considered as acceptance to participate.

Patient data was obtained from HDF digital database of IRCCS Policlinico "San Matteo" under permission of Hospital Health Management. According to the hospital procedures, all admitted patients sign an informed consent allowing the use of their anonymized data for statistical and research purposes.

We performed a data mining process to link discharge events and subsequent ER visits in Emergency Department, using the national identification number (codice fiscale) as a unique identifier. We considered all Medical Ward discharges from January $1^{\text {st }}, 2013$ to May $31^{\text {st }}, 2017$ and urgent readmission in any ward of the same hospital in the 30 days after discharge.

Readmission events were identified through the extraction of digital charts of the Emergency Department of the IRCCS Policlinico "San Matteo" Foundation, which is the only Emergency Department in the city of Pavia (approximately 75,000 inhabitants) and the referral hospital for the entire province of Pavia (approximately 500,000 inhabitants).

We defined "index cases" all the ordinary discharges as defined above and "readmissions" all the index cases that, within 30 days after a discharge, had an ER access whose outcome was "hospitalization" or "transferred"; even the latter, in fact, always identifies a direct hospitalization in another hospital.

We obtained the same readmission data for the subgroup of patient discharged with Diagnosis Related Group (DRG) 127 (Heart failure and Shock), the most frequent DRG in General Medicine in our hospital. 
Descriptive statistics were obtained for all the variables. Mean and standard deviation for quantitative variables were used, if normally distributed (Shapiro test), otherwise median and interquartile range were used.

The correlations between two quantitative variables were analyzed with the Pearson correlation coefficient.

The association between the Empathy scores (with each of the two scales) and the readmission rate of each physician was weighted for the number of cases (HDFs) of each doctor and was evaluated with multiple linear regression models, in order to consider the influence of the following factors associated with readmission:

- average Relative Weight (RW, a cost estimate of the index admission event) of the index patients discharged by each doctors; [22]

- average age of the patients of each doctor;

- patient sex;

- age of the doctor who has discharged the patient.

The results were expressed both as "correlation coefficients" (with related 95\% Confidence Intervals) and as BETA coefficients.

For quantitative variables, the "correlation coefficients" express the average change in the readmission rate for each year of age (of doctors or patients), or for each average RW point of hospitalizations. For qualitative variables, the "correlation coefficients" expresses the average change in the readmission rate for each mode of presentation of the study variable (in this study: males vs. females). The significance (p) of the coefficients was calculated by weighing the correlation for the number of index cases of each physician.

The standardized BETA coefficient was obtained to compare the relative importance of each coefficient in the regression model.[23]

For the purposes of correction, the average duration of hospitalization for each doctor was not used, as this is always strongly correlated with RW.

All tests are two-tailed, and the level of significance chosen was the usual one of $5 \%$. The analyzes were performed with the Stata software version 15.0.[24]

\section{RESULTS}

Twenty-tree envelopes were distributed with the JSE and ECQ questionnaires to General Medicine doctors who had discharged patients between 2013 and 2017. The questionnaires were distributed before knowing how many ordinary discharge procedures had been performed by each doctor: therefore, the envelopes were also handed to doctors who discharged less than 100 patients in those years. This exclusion criterion was applied subsequently, during the data processing.

To the statistician, responsible for the anonymization of the data, 22 of the 23 envelopes were returned with the completed questionnaires (96\%). All 22 returned questionnaires were fully completed.

Of the 22 participating physicians 10 were females. We measured a greater empathy in the female gender, in accordance with previous studies.[25] However, the difference is not statistically significant.

Table 1 shows the mean and median scores, in both empathy scales, in doctor's gender subgroups:

Mean age of participant doctors was $56 \pm 8$ years. There was no significant difference $(p=0,418)$ in age between male ( $55 \pm 8$ years) and female doctors $(58 \pm 9$ years). Younger doctors tended to have higher empathy scores in both JSE and ECQ scales ( $\mathrm{R}^{2} 0.639$ and 0.702 respectively), but the difference was not significant (dividing on median age $\mathrm{p}=0.217$ and $\mathrm{p}=0.113$ respectively).

General Medicine departments of the IRCCS Policlinico "San Matteo" Foundation discharged 8172 patients from January $1^{\text {st }}, 2013$ to May $31^{\text {st }}, 2017$. 
Of the total of patients, only the 4881 cases discharged at home were taken into consideration of which $49.7 \%$ were males; the characteristics of the population under analysis are presented in Table 2.

Male doctors discharged $46.6 \%$ of the patients.

Of these 4881 home discharges 4280 cases were selected as index cases, after exclusion of the cases of the doctors excluded by design (seven doctors who performed less than 100 ordinary discharges during the period under analysis).

The 4280 index cases were thus discharged by 15 doctors (of which 7 were women). The patient exclusion process is summarized in Figure 1.

In the 30 days following discharge, 716 index cases (16.7\%) had at least one access in the emergency department and $383(8.9 \%)$ were rehospitalized after ER evaluation.

The trend of ER access and readmission within 30 days from index discharge is shown in Figure 2.

Several factors were related to readmission:

- Relative Weight of the index case;

- duration of the hospitalization;

- age of the patient.

Relative Weight was significantly higher in rehospitalized compared to non-rehospitalized patients (1.211 vs 1.087, $\mathrm{p}<0.0001)$.

Readmitted patients had a longer average duration of index hospitalization, compared with non-readmitted patients (13.0 vs 11.5 days, $\mathrm{p}<0.0001)$.

Patients who are rehospitalized are significantly older than those who are not readmitted. (76.68 vs 74.72 , $\mathrm{p}=0.0078)$.

There was no significant correlation between the gender of patients and their tendency to be rehospitalized. ( $\mathrm{M} 8.9 \%$ vs $\mathrm{F} 8.7 \%, \mathrm{p}=0.791$ ). The relationship between the age of the physicians and the readmission of the patients was as well not significant. (readmitted $57.89 \mathrm{y}$, non readmitted 58.07, $\mathrm{p}=0.674$ ).

Also the relationship between the gender of the doctor and the rate of readmission had not any significance. (M $9.5 \%$ vs $\mathrm{F} 8.5 \%, \mathrm{p}=0.621$ ).

We assessed by logistic regression the correlation between the empathy score and the 30-day readmission rate of each of the 15 physicians whose data were evaluable. The correlation was adjusted for the following characteristics of each doctor: mean age of his patients; sex of his patients; average relative weight of his cases; age of the doctor himself. Correlation was weighted by the number of ordinary discharge procedures performed by each doctor.

We observed an inverse correlation between the empathy score and the readmission rate both using the JSE scale and the ECQ scale with respectively a coefficient -0.027 and -0.004 , and an $\mathrm{R}^{2} 0.181$ and 0.165 . The correlation is highly significant $(\mathrm{p}<0.001)$ for JSE scale and not significant for the ECQ scale $(\mathrm{p}=0.904$, although it was significant in univariate analysis $\mathrm{p}=0.016)$; correlation data scatter and regression lines are shown in Figure 3.

We measured the BETA value of each independent variable. BETA value indicates how each of the factors considered in the analysis can, either positively (patient age, patient sex, RW of cases), or negatively (doctor's age, empathy score) affect the occurrence of readmission.

Considering a regression model with the following independent variables - JSE score, age and sex of patients, RW of index admission and age of the physician - about one fifth (21\%) of readmissions is attributable to the empathy variable measured by JSE. 
We performed the same type of regression analysis as described above, considering only the 590 index cases of DRG 127 (Heart failure and Shock), i.e. the one with the highest number of readmission (62 or 10,5\%) andat the same time the most frequent DRG in Internal Medicine.

In the context of DRG 127, an inverse correlation emerged between the physician's empathy, and the readmission rate both on the JSE scale and on the ECQ scale with respectively coefficient -0.032 and $-0.098, \mathrm{R}^{2}$ 0.303 and $0.326, p=0.050$ and $p<0.001$; the correlation data scatter of DRG 127 is shown in Figure 4.

\section{DISCUSSION AND CONCLUSIONS}

This study demonstrates a significant correlation between the levels of empathy of Internists operating in hospital wards and the readmission rate of their patients within 30 days of discharge. This correlation remains significant after adjustment (for age and sex of the patients, for the average RW of the cases and for the age of the doctor) only using JSE measurements of empathy a tool validated specifically for the health sector); the correlation appears not significant using ECQ (a tool validated for use in the general population), whose italian translation has not been previously used. For each correlation we found rather low R-square scores, as one could expect in a study with low numbers of participants and variables with wide range of values.

The same results appear stronger considering only index cases with Heart failure (DRG 127, the most frequent in Internal Medicine), a disease where the importance of the interaction between patient and healthcare professionals appears to play a substantial role.[26] In this subgroup of patients also ECQ scores appear strongly related to the outcome, also after adjustment.

Between the Internist and the hospitalized patient there is a care relationship that can be as intense as the one between the Family Doctor and his patients. A relationship based on repeated clinical contacts, interviews and visits to establish diagnosis, to adjust therapy, to educate and make the patient aware of his illness and to plan discharge and follow up. Sometimes this process occurs directly with the patient, sometimes it happens largely with his caregivers, but it is intuitive that the Internist's empathy could have a strong influence on the patient's understanding and awareness, [27] on determining a strong therapeutic alliance,[28] in empowering the patient[29-31] and improving the management of the emotions of both the patient and his family. [32, 33] This study is therefore the first objective demonstration of the clinical relevance that empathy can have in an Internal Medicine ward, similarly to what has already been demonstrated in Family Medicine. $[3,4]$ It is true that other intermediates such as nurses, resident physicians and colleagues can affect the patient emotions and his understanding of disease and treatment, but the hypothesis that the doctor-patient relation during an average of twelve days of hospitalization has a therapeutic role, seems to us reasonable.

The yield of empathy on the outcome of the patients appears to be as big as medical interventions: for example, in patients with Heart failure (DRG 127) a difference in JSE score of 30 points appears to have the potential to reduce the readmission rate absolute value by approximately 1-2\%: an absolute risk reduction considered worth a pharmacological intervention.[18] The debate on the possibility of modulating trainees and doctors Empathy through training and education is still open.[34-36] We speculate that interventions aimed to increase hospital doctors' empathy could have a big impact on important outcomes for the patients.

Elements supporting the validity of these results include the fact that the study was conducted on a large population of patients, using complete data from accurate databases. In addition, the response rate of the Internists to whom the study was proposed was almost complete and an effective anonymization process likely reduced the occurrence of response bias. Furthermore, the results are built on readmission rate, a robust outcome measure widely used in clinical trials.

O the other hand the observational and retrospective nature of the study cannot demonstrate causality, but only correlations deserving further investigation. Furthermore, our data on readmission doesn't include those of other hospitals, though we estimate that access to other minor emergency departments, after an index discharge from our teaching hospital is a rare event: our emergency rescue service favors patient's return to 
the same hospital that recently discharged him/her.[37] Moreover, our data does not consider deaths outside the hospital but we estimate this kind of events now very rare in the highly urbanized area of Pavia province.

A further limitation of the study concerns the empathy evaluation method which, by its nature, in addition to being self-assessed, does not consider possible variations of empathy over the long period considered.

Lastly, our Italian translation of ECQ has not been validated. This fact is a limitation of the study, because without a proper psychometric assessment, these tests may lose their validity if translated into another language; a good correlation between the results obtained with the two scales reassures on the validity of the results.

The small number of doctors involved reduces the validity of the study and probably causes low correlation values; further studies, on larger populations, are necessary to confirm these findings, that could have a great relevance for the training of physicians, both in medical school and in continuing medical education.

\section{BIBLIOGRAPHY}

1. Hippocrates (1923) Hippocrates. Volume II: on decorum and the physician. Heinemann, London

2. Hojat M (2007) Empathy in patient care : antecedents, development, measurement, and outcomes. Springer

3. Hojat M, Louis DZ, Markham FW, et al (2011) Physicians' empathy and clinical outcomes for diabetic patients. Acad Med 86:359-364. https://doi.org/10.1097/ACM.0b013e3182086fe1

4. Del Canale S, Louis DZ, Maio V, et al (2012) The relationship between physician empathy and disease complications: An empirical study of primary care physicians and their diabetic patients in Parma, Italy. Acad Med 87:1243-1249. https://doi.org/10.1097/ACM.0b013e3182628fbf

5. Rakel D, Barrett B, Zhang Z, et al (2011) Perception of empathy in the therapeutic encounter: Effects on the common cold. Patient Educ Couns 85:390-397. https://doi.org/10.1016/j.pec.2011.01.009

6. Kelley JM, Kraft-Todd G, Schapira L, et al (2014) The Influence of the Patient-Clinician Relationship on Healthcare Outcomes: A Systematic Review and Meta- Analysis of Randomized Controlled Trials. PLoS One 9:101191. https://doi.org/10.1371/journal.pone.0094207

7. Ogle J, Bushnell JA, Caputi P (2013) Empathy is related to clinical competence in medical care. Med Educ 47:824-831. https://doi.org/10.1111/medu.12232

8. Mercer SW, Reynolds WJ (2002) Empathy and quality of care. Br J Gen Pract 52 Suppl:S9-12

9. Hojat M, Louis DZ, Maio V, Gonnella JS (2013) Editorial: Empathy and Health Care Quality. Am J Med Qual 28:6-7. https://doi.org/10.1177/1062860612464731

10. Bellet PS, Maloney MJ (1991) The Importance of Empathy as an Interviewing Skill in Medicine. JAMA J Am Med Assoc 266:1831-1832. https://doi.org/10.1001/jama.1991.03470130111039

11. Lelorain S, Bredart A, Dolbeault S, Sultan S (2012) A systematic review of the associations between empathy measures and patient outcomes in cancer care. Psychooncology 21:1255-1264. https://doi.org/10.1002/pon.2115

12. Kim SS, Kaplowitz S, Johnston M V. (2004) The effects of physician empathy on patient satisfaction and compliance. Eval Heal Prof 27:237-251. https://doi.org/10.1177/0163278704267037

13. William Osler (1899) Address to the students of the Albany Medical College. In: Albany Medical Annals. pp 20:307-309

14. Zamir D, Zamir M, Reitblat T, et al (2006) Readmissions to hospital within 30 days of discharge from the internal medicine wards in southern Israel. Eur J Intern Med 17:20-23. https://doi.org/10.1016/j.ejim.2005.10.004 
15. Hansen LO, Young RS, Hinami K, et al (2011) Interventions to reduce 30-day rehospitalization: A systematic review. Ann. Intern. Med. 155:520-528

16. De Giorgi A, Boari B, Tiseo R, et al (2016) Hospital readmissions to internal medicine departments: A higher risk for females? Eur Rev Med Pharmacol Sci 20:4557-4564

17. Jencks SF, Williams M V., Coleman EA (2009) Rehospitalizations among Patients in the Medicare Fee-for-Service Program. N Engl J Med 360:1418-1428. https://doi.org/10.1056/NEJMsa0803563

18. Borer JS, Bohm M, Ford I, et al (2015) Effect of Ivabradine on Early Readmissions After Hospitalization for Worsening Heart Failure. JACC Hear Fail 3:268-269. https://doi.org/10.1016/j.jchf.2014.12.004

19. Hojat M (2016) Empathy in Health Professions Education and Patient Care

20. Batchelder L, Brosnan M, Ashwin C (2017) The development and validation of the empathy components questionnaire (ECQ)

21. Nederhof AJ (1985) Methods of coping with social desirability bias: A review. Eur J Soc Psychol 15:263-280. https://doi.org/10.1002/ejsp.2420150303

22. Ufficio Della Programmazione Sanitaria (2010) Rapporto annuale sull'attivita di ricovero ospedaliero

23. Freedman DA (2009) Statistical Models: Theory and Practice. Cambridge University Press

24. StataCorp (2017) Stata Statistical Software: Release 15

25. Hojat M, DeSantis J, Shannon SC, et al (2018) The Jefferson Scale of Empathy: a nationwide study of measurement properties, underlying components, latent variable structure, and national norms in medical students. Adv Heal Sci Educ. https://doi.org/10.1007/s10459-018-9839-9

26. Currie K, Strachan PH, Spaling M, et al (2015) The importance of interactions between patients and healthcare professionals for heart failure self-care: A systematic review of qualitative research into patient perspectives. Eur J Cardiovasc Nurs 14:525-535. https://doi.org/10.1177/1474515114547648

27. Braddock CH, Edwards KA, Hasenberg NM, et al Informed decision making in outpatient practice: time to get back to basics. JAMA 282:2313-20

28. Fuertes JN, Mislowack A, Bennett J, et al (2007) The physician-patient working alliance. Patient Educ Couns 66:29-36. https://doi.org/10.1016/j.pec.2006.09.013

29. Glasgow RE, Whitesides H, Nelson CC, King DK (2005) Use of the Patient Assessment of Chronic Illness Care (PACIC) with diabetic patients: relationship to patient characteristics, receipt of care, and self-management. Diabetes Care 28:2655-61

30. Williams GC, Rodin GC, Ryan RM, et al (1998) Autonomous regulation and long-term medication adherence in adult outpatients. Health Psychol 17:269-76

31. Williams GC, McGregor HA, King D, et al (2005) Variation in perceived competence, glycemic control, and patient satisfaction: relationship to autonomy support from physicians. Patient Educ Couns 57:39-45. https://doi.org/10.1016/j.pec.2004.04.001

32. Salmon P (2006) The potentially somatizing effect of clinical consultation. CNS Spectr 11:190-200

33. H. R, Schofield P, Cockburn J, et al (2005) How to recognize and manage psychological distress in cancer patients. Eur J Cancer Care (Engl) 14:7-15. https://doi.org/10.1111/j.1365-2354.2005.00482.x

34. Riess H, Kelley JM, Bailey RW, et al (2012) Empathy Training for Resident Physicians: A Randomized Controlled Trial of a Neuroscience-Informed Curriculum. J Gen Intern Med 27:1280-1286. https://doi.org/10.1007/s11606-012-2063-z 
35. Chen P-J, Huang C-D, Yeh S-J (2017) Impact of a narrative medicine programme on healthcare providers' empathy scores over time. BMC Med Educ 17:108. https://doi.org/10.1186/s12909-017-0952-x

36. Hojat M (2009) Ten approaches for enhancing empathy in health and human services cultures. J Health Hum Serv Adm 31:412-50

37. Azienda Regionale Emergenza Urgenza. http://www.areu.lombardia.it/web/home/carta-dei-servizi. Accessed 2 Feb 2018

TABLES

\begin{tabular}{llllll}
\hline & & All $(\mathrm{n}=22)$ & Male $(\mathrm{n}=12)$ & Female $(\mathrm{n}=10)$ & $\mathrm{p}$ \\
\hline JSE & mean \pm sd & $110 \pm 14$ & $108 \pm 14$ & $113 \pm 15$ & 0.462 \\
& median (IQR) & $107(97.75 ; 123.75)$ & $107(101.5 ; 120)$ & $112.5(97.75 ; 127.25)$ & \\
ECQ & mean \pm sd & $86 \pm 10$ & $84 \pm 10$ & $88 \pm 11$ & 0.305 \\
& median (IQR) & $89(79.5 ; 91.75)$ & $89(77.75 ; 90.25)$ & $89(83.5 ; 92)$ & \\
\hline
\end{tabular}

Table 1 values of empathy of doctors; JSE = Jefferson Scale of Empathy, ECQ = Empathy Components Questionnaire, IQR = interquartile range

\begin{tabular}{lllll}
\hline & Mean & Stand. Dev. & Median & IQR \\
\hline Patient's age (y) & 74.7 & 14.6 & 78 & $68 ; 85$ \\
Relative Weight & 1.086 & 0.46 & 1.050 & $0.799 ; 1.26$ \\
Duration of hospitalization (d) & 11.8 & 6.8 & 10 & $7 ; 14$ \\
\hline
\end{tabular}

Table 2 characteristics of patients; $\mathrm{IQR}=$ interquartile range. $\mathrm{y}=$ years, $\mathrm{d}=$ days

\section{FIGURE CAPTIONS}

Fig. 1 Patient exclusion process for index case eligibility

Fig. 2 Trend of ER access (grey columns) and readmission events (black columns) in the first 30 days after index discharge

Fig. 3

a Correlation between the Empathy Components Questionnaire score and the 30-day readmission rate of the 15 physicians whose data were evaluable (all DRGs included); each grey triangle represents one doctor and the dotted line is the linear correlation; the correlation is adjusted for the following characteristics of each doctor: mean age of his patients, sex of his patients, average relative weight of his cases, age of the doctor himself; the correlation is weighted by the number of ordinary discharge procedures performed by each doctor; ECQ = Empathy Components Questionnaire

b Correlation between the Jefferson Scale of Empathy score and the 30-day readmission rate of the 15 physicians whose data were evaluable (all DRGs included); each black dot represents one doctor and the solid line is the linear correlation; the correlation is adjusted for the following characteristics of each doctor: mean age of his patients, sex of his patients, average relative weight of his cases, age of the doctor himself; the correlation is weighted by the number of ordinary discharge procedures performed by each doctor; JSE $=$ Jefferson Scale of Empathy

Fig. 4

a Correlation between the Empathy Components Questionnaire score and the 30-day readmission rate of the 15 physicians in DRG 127 subgroup; each grey triangle represents one doctor and the dotted line is the 
linear correlation; the correlation is adjusted for the following characteristics of each doctor: mean age of his patients, sex of his patients, average relative weight of his cases, age of the doctor himself; the correlation is weighted by the number of ordinary discharge procedures performed by each doctor; ECQ = Empathy Components Questionnaire

b Correlation between the Jefferson Scale of Empathy score and the 30-day readmission rate of the 15 physicians in DRG 127 subgroup; each black dot represents one doctor and the solid line is the linear correlation; the correlation is adjusted for the following characteristics of each doctor: mean age of his patients, sex of his patients, average relative weight of his cases, age of the doctor himself; the correlation is weighted by the number of ordinary discharge procedures performed by each doctor; JSE = Jefferson Scale of Empathy

\section{Hosted file}

Fig.1 Empathy IJCP.docx available at https://authorea.com/users/318134/articles/448083correlation-between-attending-physician-s-empathy-and-readmission-rate-in-internalmedicine

\section{Hosted file}

Fig.2 Empathy IJCP.docx available at https://authorea.com/users/318134/articles/448083correlation-between-attending-physician-s-empathy-and-readmission-rate-in-internalmedicine

\section{Hosted file}

Fig.3 Empathy IJCP.docx available at https://authorea.com/users/318134/articles/448083correlation-between-attending-physician-s-empathy-and-readmission-rate-in-internalmedicine

\section{Hosted file}

Fig.4 Empathy ICJP.docx available at https://authorea.com/users/318134/articles/448083correlation-between-attending-physician-s-empathy-and-readmission-rate-in-internalmedicine 